Research Article

\title{
Comparison of Performance of Equations for Estimated Glomerular Filtration Rate in Chinese Patients with Biopsy-Proven Diabetic Nephropathy
}

\author{
Yiting Wang, ${ }^{1}$ Junlin Zhang, ${ }^{1}$ Geer Teng, ${ }^{2}$ Yucheng Wu, ${ }^{1}$ Qianqian Han, ${ }^{1}$ Hanyu Li, ${ }^{1}$ \\ Tingli Wang, ${ }^{1}$ and Fang Liu ${ }^{1}{ }^{1}$ \\ ${ }^{1}$ Division of Nephrology, West China Hospital of Sichuan University, Chengdu 610041, China \\ ${ }^{2}$ The Faculty of Social Development and Western China Development Studies, Sichuan University, China \\ Correspondence should be addressed to Fang Liu; liufangfh@163.com
}

Received 1 July 2019; Accepted 28 August 2019; Published 15 September 2019

Academic Editor: Hubertus Himmerich

Copyright (C) 2019 Yiting Wang et al. This is an open access article distributed under the Creative Commons Attribution License, which permits unrestricted use, distribution, and reproduction in any medium, provided the original work is properly cited.

\begin{abstract}
Background. The performance of various equations for estimated glomerular filtration rate (eGFR) in patients with diabetes remains controversial. We aimed to evaluate the performance of equations for eGFR in Chinese patients with diabetic nephropathy (DN). Methods. This is a retrospective study included in 308 patients with type 2 diabetes and biopsy-proven DN who were followed up at least one year. eGFR was calculated using chronic kidney disease epidemiology (CKD-EPI) equations

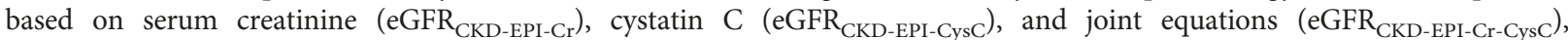
respectively. End-stage kidney disease was defined by initiation of renal replacement therapy. The eGFR concordance between equations was assessed by Bland-Altman plots. Log-rank and multivariable logistic regression were employed to evaluate the performance of equations. Results. Overall, the proportion of patients with eGFR $<60 \mathrm{~mL} / \mathrm{min} / 1.73 \mathrm{~m}^{2}$ was $53 \%, 70 \%$, and $61 \%$

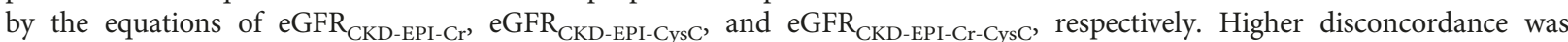
observed between equations when eGFR $>60 \mathrm{~mL} / \mathrm{min} / 1.73 \mathrm{~m}^{2}$. Compared with eGFR CKD-EPI-Cr, $39 \%$ of patients were reclassified

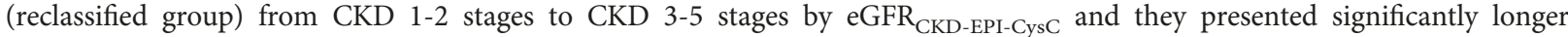
diabetic duration, heavier proteinuria, advanced pathological lesions, and poorer kidney outcomes. Multivariable logistic regression indicated cystatin $\mathrm{C}$ was independently associated with advanced glomerular classifications. Conclusion. eGFR equations incorporating cystatin $\mathrm{C}$ are superior to eGFR based on creatinine alone for detecting kidney injury in the early stage. The independent association between cystatin $\mathrm{C}$ and glomerular classifications might contribute to it.
\end{abstract}

\section{Introduction}

The past few decades have witnessed a marked increasing prevalence of type 2 diabetes, especially in China, and the global prevalence of microvascular and macrovascular complications associated with diabetes increases dramatically $[1,2]$. Diabetic nephropathy (DN) has become the leading cause of end-stage kidney disease (ESKD) worldwide $[3,4]$. The utilization of renin-angiotensin-aldosterone system blockers and improvements in glycemic, blood pressure, and lipid control slow the progression of chronic kidney disease (CKD) to a degree [5]. Indeed, glomerular filtration rate (GFR) guides the clinical management of CKD and is an independent predictor of kidney injury, all-cause/cardiovascular mortality, and kidney failure [6]. Therefore, accurate estimation of GFR to identify CKD and predict kidney outcome is highlighted.

The Kidney Disease Improving Global Outcomes (KDIGO) 2012 Clinical Practice Guideline for the Evaluation and Management of CKD [7] recommends initial use of 2009 CKD-Epidemiology Collaboration equation based on serum creatinine (eGFR $\mathrm{CKD}_{\text {-EPI-Cr}}$ ) instead of the Modification of Diet in Renal Disease equation. They also suggest use of the 2012 CKD-EPI equations (eGFR ${ }_{\text {CKD-EPI-CysC }}$, $\mathrm{eGFR}_{\text {CKD-EPI-Cr-CysC }}$ ) to confirm kidney function when cystatin C has been measured, particularly for patients 
with $\mathrm{eGFR}_{\mathrm{Cr}}$ of $45-59 \mathrm{~mL} / \mathrm{min} / 1.73 \mathrm{~m}^{2}$ who do not have markers of kidney damage. However, the performance of various equations in CKD cohorts remains controversial due to serum creatinine is influenced by age, muscle mass, sex, and race; cystatin $\mathrm{C}$ level is affected by ages, body mass index, diabetes, and inflammation. Patients with $\mathrm{DN}$ are recognized as a special community in CKD. Also, the implications and predictive potential of different equations in patients with $\mathrm{DN}$ have yet to be elucidated. The objective of the study was to compare the performance for detecting kidney injury of equations of eGFR CKD-EPI-Cr $_{\text {, }}$ eGFR $_{\text {CKD-EPI-CySC }}$, and eGFR ${ }_{\text {CKD-EPI-Cr-CysC }}$ in patients with $\mathrm{DN}$ in a single center in Southwest China.

\section{Method}

2.1. Study Population. This is a retrospective cohort study. From November 2003 to March 2018, a total of 308 Chinese patients with type 2 diabetes mellitus (T2DM) and biopsy-proven DN in West China Hospital of Sichuan University were recruited and followed up for at least one year by routine clinical visits. Patients with T2DM and proteinuria $>0.5 \mathrm{~g} / 24 \mathrm{~h}$ or eGFR decline were indicated to receive kidney biopsy in our hospital. The diagnoses of T2DM and DN were based upon the criteria recommended by the American Diabetes Association (ADA) in 2018 [8] and the Renal Pathology Society in 2010 [9]. The ESKD was defined by initiation of renal replacement therapy (hemodialysis, peritoneal dialysis, or kidney transplantation). Patients who had malignances, nondiabetic renal disease (NDRD), and NDRD+DN were excluded from the study.

The protocol of study was approved by the ethics committee of West China Hospital of Sichuan University and conducted based on the principles of the Declaration of Helsinki; written informed consents were obtained at the time of biopsy from all the patients.

2.2. Clinical and Pathological Characteristics. Diabetic history, data including physical examinations (body mass index, blood pressure, and examination of diabetic retinopathy) and laboratory tests $(\mathrm{HbAlc}$, 24-hour protein excretion, serum creatinine, serum cystatin $\mathrm{C}$, serum lipid), were collected at the time of kidney biopsy from the hospital information system. Creatinine was measured using Jaffe's assay. Serum creatinine value was calibrated to isotope dilution mass spectrometry (IDMS). Serum cystatin C was measured using an automated particle-enhanced immunoturbidimetric method. Blood samples were collected after 12 hours of fasting in all the patients [10]. Pathological lesions were routinely assessed under light and electron microscopy by at least two nephropathologists according to criteria proposed by the Renal Pathology Society in 2010 [9].

2.3. Statistical Analysis. GFR was estimated using the equations [11] of eGFR ${ }_{\text {CKD-EPI-Cr }}$, eGFR CKD-EPI-CysC $_{\text {, and }}$ eGFR $_{\text {CKD-EPI-Cr-CysC }}$, respectively. The CKD stages $1,2,3 \mathrm{a}$, $3 \mathrm{~b}, 4$, and 5 were categorized by eGFR $(\geq 90,60-90,45-60$, $30-45,15-30, \leq 15 \mathrm{~mL} / \mathrm{min} / 1.73 \mathrm{~m}^{2}$ ) [12]. The term "reduced kidney function" indicated eGFR $<60 \mathrm{~mL} / \mathrm{min} / 1.73 \mathrm{~m}^{2}$. The bias (mean difference) between each two equations was assessed by Bland-Altman plots. The differences in variables were analyzed appropriately by Student's $t$-test, the MannWhitney test, or the chi-square test. Kidney outcomes were compared using the log-rank test and demonstrated by the Kaplan-Meier curves method. The association between variables was analyzed by multivariable logistic regression. All analyses were conducted using SPSS software 22.0 and GraphPad Prism 7.0, and a two-sided $P$ value of less than 0.05 was considered statistically significant.

\section{Results}

3.1. Baseline Demographic Clinical and Pathological Characteristics. A total of 308 patients with biopsy-proven DN were enrolled in the current study (Table 1). Among them, $69.5 \%$ of patients were male and the mean age was $51.4 \pm 9.7$ years old. The mean duration of diabetes was $97.8 \pm 70.3$ months. $44.8 \%$ and $86.0 \%$ of patients have diabetic retinopathy and hypertension, respectively. The mean serum creatinine and cystatin $\mathrm{C}$ were $130 \pm 65 \mu$ $\mathrm{mol} / \mathrm{L}$ and $1.61 \pm 0.60 \mathrm{mg} / \mathrm{L}$, respectively. The median (interquartile range) initial proteinuria was 4.3 (2.0-7.8) $\mathrm{g} / 24$ hours. The thyroid levels were in normal range. The mean GFR was higher when estimated using the CKDEPI-Cr $\left(62.6 \pm 28.7 \mathrm{~mL} / \mathrm{min} / 1.73 \mathrm{~m}^{2}\right)$ than using CKDEPI-CysC $\left(50.3 \pm 23.3 \mathrm{~mL} / \mathrm{min} / 1.73 \mathrm{~m}^{2}\right)$ and CKD-EPICr-CysC $\left(54.6 \pm 24.9 \mathrm{~mL} / \mathrm{min} / 1.73 \mathrm{~m}^{2}\right) .81 .5 \%$ of patients received RAAS inhibitors, $72.1 \%$ of patients received insulin therapy, and $57.5 \%$ of patients received statins.

All the patients in the study underwent kidney biopsy. Glomerular lesions were classified as follows. Seventeen patients had glomerular basement membrane thickening only and were classified as class I. 104 patients had mild or severe mesangial expansion, but without nodular sclerosis (Kimmelstiel-Wilson lesion), and were classified as class II. 140 patients who did not meet the criteria of class IV with at least one convincing Kimmelstiel-Wilson lesion were classified as class III. 47 patients with global glomerular sclerosis in $\geq 50 \%$ of glomeruli were classified as class IV. For the IFTA score, 10, 138, 127, and 33 patients were scored as 0 , 1,2 , and 3 , respectively. For interstitial inflammation, 20, 233 , and 50 patients were scored as 0,1 , and 2, respectively. For arteriolar hyalinosis, 34, 162, and 112 patients were scored as 0,1 , and 2 , respectively.

During a median follow-up period of 20 (13-33) months, a total of $131(42.5 \%)$ patients reached ESKD.

\subsection{CKD Stages Categorized Using Different Equations.}

Figure 1 shows the proportion of CKD stages categorized using the different equations. Overall, more patients tend to be categorized into advanced CKD stages by eGFR $\mathrm{CKD}_{\text {EPI- }}$ CysC $_{\text {and }}$ aGFR CKD-EPI-Cr-CysC $_{\text {compared with eGFR }}$ CKD-EPI$\mathrm{Cr}$. Approximately half of patients (47\%) were included in CKD 1 and CKD 2 stages when using equation of eGFR $_{\text {CKD-EPI-Cr }}$, but only $30 \%$ of patients in CKD 1 and 2 stages when using equation of $\mathrm{eGFR}_{\mathrm{CKD}-\mathrm{EPI}-\mathrm{CySC} \text {. }}$ On the contrary, 62 patients were categorized into CKD $3 \mathrm{~b}$ stage and 39 patients were in CKD 4 stage when using eGFR CKD-EPI-Cr , 
TABLE 1: Baseline demographic, clinical, and pathological characteristics.

\begin{tabular}{|c|c|}
\hline Variables & Patients $(n=308)$ \\
\hline Age (years) & $51.4 \pm 9.7$ \\
\hline Gender (male) & $214(69.5)$ \\
\hline Duration of diabetes (months) & $97.8 \pm 70.3$ \\
\hline Diabetic retinopathy (\%) & $138(44.8)$ \\
\hline Body mass index $\left(\mathrm{kg} / \mathrm{m}^{2}\right)$ & $25.78 \pm 3.78$ \\
\hline Hypertension (\%) & $265(86.0)$ \\
\hline Systolic blood pressure (mmHg) & $145 \pm 23$ \\
\hline Diastolic blood pressure (mmHg) & $86 \pm 13$ \\
\hline HbA1C (\%) & $7.3(6.3-8.5)$ \\
\hline Initial proteinuria (g/24h) & $4.3(2.0-7.8)$ \\
\hline Serum creatinine $(\mu \mathrm{mol} / \mathrm{L})$ & $130 \pm 65$ \\
\hline Cystatin C (mg/L) & $1.61 \pm 0.60$ \\
\hline Triglyceride (mmol/L) & $2.19 \pm 1.80$ \\
\hline Total cholesterol (mmol/L) & $5.22 \pm 1.60$ \\
\hline $\begin{array}{l}\text { Thyroid-stimulating hormone } \\
\text { (mU/L) }\end{array}$ & $3.15(1.96-5.60)(n=155)$ \\
\hline Free triiodothyronine 3 (pmol/L) & $3.92(3.39-4.41)(n=151)$ \\
\hline Free triiodothyronine $4(\mathrm{pmol} / \mathrm{L})$ & $14.95(13.17-16.61)(n=154)$ \\
\hline eGFR $_{\text {CKD-EPI-Cr }}$ & $62.6 \pm 28.7$ \\
\hline $\mathrm{eGFR}_{\mathrm{CKD} \text {-EPI-CysC }}$ & $50.3 \pm 23.3$ \\
\hline $\mathrm{eGFR}_{\text {CKD-EPI-Cr-CysC }}$ & $54.6 \pm 24.9$ \\
\hline RAAS inhibitors (\%) & $251(81.5)$ \\
\hline Insulin therapy (\%) & $222(72.1)$ \\
\hline Statins (\%) & $177(57.5)$ \\
\hline \multicolumn{2}{|l|}{ Glomerular class } \\
\hline I & 17 \\
\hline IIa & 75 \\
\hline $\mathrm{IIb}$ & 29 \\
\hline III & 140 \\
\hline IV & 47 \\
\hline \multicolumn{2}{|l|}{$\begin{array}{l}\text { Interstitial fibrosis and } \\
\text { tubular atrophy }\end{array}$} \\
\hline 0 & 10 \\
\hline 1 & 138 \\
\hline 2 & 127 \\
\hline 3 & 33 \\
\hline \multicolumn{2}{|l|}{ Interstitial inflammation } \\
\hline 0 & 20 \\
\hline 1 & 233 \\
\hline 2 & 55 \\
\hline \multicolumn{2}{|l|}{ Arteriolar hyalinosis } \\
\hline 0 & 34 \\
\hline 1 & 162 \\
\hline 2 & 112 \\
\hline Reach end-stage kidney disease & 131 \\
\hline $\begin{array}{l}\text { Median kidney survival time } \\
\text { (months) }\end{array}$ & $20(13-33)$ \\
\hline
\end{tabular}

Data are presented as the mean \pm standard and the median with range or counts and percentages.

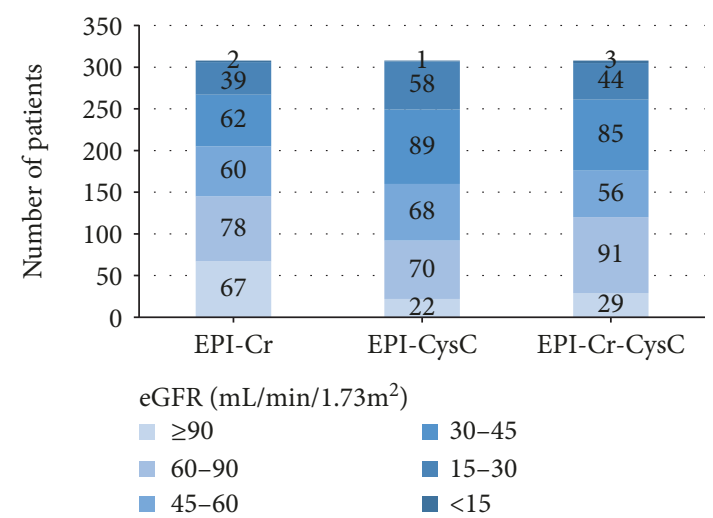

FIGURE 1: CKD stages categorized using different equations eGFR $\left(\mathrm{mL} / \mathrm{min} / 1.73 \mathrm{~m}^{2}\right)$. CKD stage 1: eGFR $\geq 90 \mathrm{~mL} / \mathrm{min} / 1.73 \mathrm{~m}^{2}$; CKD stage 2: eGFR $(60-90) \mathrm{mL} / \mathrm{min} / 1.73 \mathrm{~m}^{2}$; CKD stage $3 \mathrm{a}$ : eGFR (45-60) $\mathrm{mL} / \mathrm{min} / 1.73 \mathrm{~m}^{2}$; CKD stage $3 \mathrm{~b}$ : eGFR (30-45) $\mathrm{mL} / \mathrm{min} / 1.73 \mathrm{~m}^{2}$; CKD stage 4 : eGFR (15-30) $\mathrm{mL} / \mathrm{min} / 1.73 \mathrm{~m}^{2}$; CKD stage 5: eGFR $<15 \mathrm{~mL} / \mathrm{min} / 1.73 \mathrm{~m}^{2}$. More patients tend to be categorized into advanced CKD stages by eGFRCKD-EPICysC and eGFRCKD-EPI-Cr-CysC compared with eGFRCKDEPI-Cr.

but 89 and 58 patients were in CKD 3 b and 4 stages, respectively, when using eGFR $\mathrm{CKD}_{\text {-EPI-CysC. }}$

The Bland-Altman plot shown in Figure 2 revealed the bias between each two equations. Overall, high disconcordance was observed between eGFR $\mathrm{CKD}_{\mathrm{CPI}-\mathrm{Cr}}$ and $\mathrm{eGFR}_{\mathrm{CKD}}$ EPI-CysC, especially when $e G F R \geq 60 \mathrm{~mL} / \mathrm{min} / 1.73 \mathrm{~m}^{2}$. The mean bias of eGFR ${ }_{\text {CKD-EPI-Cr }}$ and eGFR $_{\text {CKD-EPI-CysC }}$ was $12.28 \pm 13.7 \mathrm{~mL} / \mathrm{min} / 1.73 \mathrm{~m}^{2}$, and upper $95 \%$ limit of agreement was $39.13 \mathrm{~mL} / \mathrm{min} / 1.73 \mathrm{~m}^{2}$, which were beyond accepted limit. The mean bias of $e_{G F R}$ CKD-EPI-Cr and eGFR $_{\text {CKD-EPI-Cr-CysC }}$ was $7.98 \pm 8.32 \mathrm{~mL} / \mathrm{min} / 1.73 \mathrm{~m}^{2}$, and the mean bias of eGFR $\mathrm{CKD}_{\text {-EPI-Cr-CysC }}$ and eGFR ${ }_{\mathrm{CKD}-\mathrm{EPI}-\mathrm{CysC}}$ was $4.29 \pm 5.64 \mathrm{~mL} / \mathrm{min} / 1.73 \mathrm{~m}^{2}$.

3.3. Reclassification to Reduced Kidney Function by $e G F R_{C K D-E P I-C y s C}$ Equation. A total of 145 patients were with $\mathrm{eGFR}>60 \mathrm{~mL} / \mathrm{min} / 1.73 \mathrm{~m}^{2}$ (CKD 1 and 2 stages) when using eGFR $\mathrm{CKD}_{\text {-EPI-Cr }}$ equation; however, 57 of them were reclassified into $\mathrm{CKD}$ 3-5 stages when using eGFR $_{\text {CKD-EPI-CysC }}$ equation (Table 2). Compared with patients who were not reclassified $(n=88)$, the reclassified group presented significantly longer diabetic duration (106.8 \pm 64.8 vs $83.4 \pm 61.6$ months), heavier proteinuria [4.2(2.3-8.8) vs $2.4(0.8-4.8) \mathrm{g} / 24 \mathrm{~h}, P=0.001]$, higher level of cystatin C $(1.45 \pm 0.20 \mathrm{vs} 1.02 \pm 0.15 \mathrm{mg} / \mathrm{L}, P<0.001)$, lower free triiodothyronine 3 [3.92 (3.46-4.35) vs 4.41 (3.92$5.10) \mathrm{pmol} / \mathrm{L}, P=0.003$ ], advanced glomerular classifications $(P<0.001)$, IFTA scores $(P<0.001)$, and interstitial inflammation $(P<0.001)$. In addition, during the follow-up, 11 $(12.5 \%)$ patients reached the end point in the not reclassified group, while $21(36.8 \%)$ patients reached the end point in the reclassified group; the kidney survival was significantly poorer by the log-rank test in the reclassified group (Figure 3(a)). Similarly, 26 patients were reclassified into $\mathrm{CKD}$ 3-5 stages using $\mathrm{eGFR}_{\mathrm{CKD}-\mathrm{EPI}-\mathrm{Cr}-\mathrm{CysC}}$ equation 

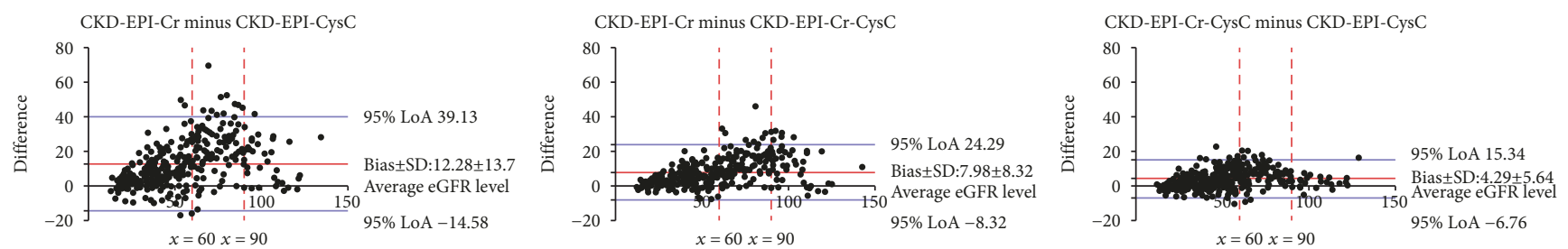

FIGURE 2: Bland-Altman plots of the difference between eGFRs. The limits of agreement (LoA) are defined as the mean difference \pm 1.96 SD of differences. The black line and the blue lines indicate mean difference and $95 \%$ LoA, respectively. The red dashed lines indicate when average eGFR are 60 and $90 \mathrm{~mL} / \mathrm{min} / 1.73 \mathrm{~m}^{2}$. High disconcordance was observed between eGFR $\mathrm{CKD}_{\text {-EPI-Cr }}$ and eGFR $\mathrm{CKD}_{\text {-EPI-CysC }}$, especially when eGFR $>60 \mathrm{~mL} / \mathrm{min} / 1.73 \mathrm{~m}^{2}$. SD: standard deviation.

from patients with $\mathrm{eGFR}_{\mathrm{CKD}-\mathrm{EPI}-\mathrm{Cr}}>60 \mathrm{~mL} / \mathrm{min} / 1.73 \mathrm{~m}^{2}$. A significantly poorer kidney outcome was observed in patients who were reclassified by eGFR $_{\text {CKD-EPI-Cr-CysC }}$ during the follow-up period (Figure 3(b)).

\subsection{The Association between Serum Creatinine and Cystatin} $C$ and Pathological Lesions. We then investigated the association between serum creatinine and cystatin $\mathrm{C}$ and pathological lesions. For glomerular classification, we defined class of III and IV as advanced lesion, and for tubular and interstitial lesions, we defined scores of 2 and 3 of IFTA as advanced lesions. We adjusted essential clinical variables including gender, age, blood pressure, diabetic duration, triglyceride, total cholesterol, and proteinuria for multivariable logistic regression. As shown in Table 3, only gender (odds ratio (OR) 0.349, 95\% confidence interval (CI) $0.174-0.700, P=0.003$ ) and cystatin C (OR 3.771, 95\% CI 1.140-12.472, $P=0.030)$ were independently associated with advanced glomerular lesions. Serum creatinine (OR 1.004, 95\% CI 0.993-1.015, P>0.05) was not independently associated with advanced glomerular lesions. However, total cholesterol (OR 1.249, 95\% CI 1.009$1.545, P=0.041$ ) and serum creatinine (OR 1.012, 95\% CI 1.002-1.023, $P=0.024)$ were independently associated with advanced tubular and interstitial injury.

\section{Discussion}

Despite decades of research and heavy public health burden associated with DN, few new biomarkers have been applied to clinical practice in recent years [13]. Albuminuria and eGFR are still essential ones to monitor kidney function and guide management for patients with DN. However, the performance of GFR estimated by different equations is still under debate [14-16]. The current study showed the distribution of CKD stages categorized by different equations in patients with kidney-biopsy DN. 39\% of patients with CKD 1 and 2 stages (by eGFR $\mathrm{CKD}_{\mathrm{EPI}-\mathrm{Cr}}$ ) were reclassified into advanced CKD stages (by eGFR $\mathrm{CKD}_{\text {-EPI-CysC }}$ ) and they had longer diabetic duration, heavier proteinuria, advanced pathological lesions, and poorer prognosis. In addition, cystatin $\mathrm{C}$, not creatinine, was independently associated with more severe glomerular classifications. Those findings suggest that equations incorporate cystatin $\mathrm{C}$ would improve the performance of detect glomerular lesions in the early stage in patients with DN.
Both serum creatinine and cystatin $\mathrm{C}$ are endogenous molecules. Serum creatinine is unstable and easily influenced by daily diet [10], secretion and reabsorption of tubular cells [17], and reduced muscle mass [18] which is common in patients with CKD [19]. Cystatin C is a low molecular basic protein, which is reabsorbed and catabolized by tubular cells completely. The serum concentrate is mainly affected by gender, obesity, diabetes, and hypertension [20, 21]. Criteria for selecting the optimal GFR estimating equation are accuracy, discrimination of kidney outcomes [22], and the population characteristic. Recently, substantial studies have evaluated the performance of different eGFR equations in the population of diabetes, $\mathrm{CKD}$, or $\mathrm{CKD}$ with diabetes, but the conclusions remain largely controversial.

In the U.S. population of noninstitutionalized civilian, the prevalence of reduced kidney function was $6.5 \%$ when estimated using equations based on creatinine, compared with $8.7 \%$ when incorporated with cystatin C [23]. Similarly, a study enrolled 778 persons with diabetes detected the prevalence of reduced kidney function was $16.5 \%$ and $22.0 \%$ using eGFR $\mathrm{Cr}_{\mathrm{r}}$ and $e \mathrm{GFR}_{\mathrm{CysC}}$, respectively. And patients with diabetes were more likely to be reclassified from preserved kidney function calculated by $\mathrm{eGFR}_{\mathrm{Cr}}$ to reduced kidney function calculated by $\mathrm{eGFR}_{\mathrm{Cys}}[20]$. However, a study included 199 diabetic patients demonstrated that both $\mathrm{eGFR}_{\mathrm{MDRD}}$ and $\mathrm{eGFR}_{\mathrm{CKD} \text {-EPI-Cr }}$ equations underestimated measured GFR (>90 mL/min $\left./ 1.73 \mathrm{~m}^{2}\right)$ [24]. Moreover, long-term GFR decline was proved to be largely underestimated in a cross-sectional and longitudinal analysis [21]. In the current study, a biopsy-proven DN cohort, $53 \%$ of patients were with reduced kidney function using eGFR $_{\text {CKD-EPI-Cr }}$ while $70 \%$ of which using eGFR ${ }_{\text {CKD-EPI-CysC }}$. Interestingly, patients who were reclassified by eGFR ${ }_{\mathrm{CKD} \text {-EPI- }}$ CysC have significantly heavier proteinuria, advanced pathological lesions, and faster progression of kidney disease than not reclassified patients, which suggested that GFFR $_{\mathrm{CKD} \text {-EPI- }}$ CysC was more sensitive to detect kidney injury and predict kidney outcomes.

However, several studies questioned the improved performance of equations based on cystatin C. A latest study with 882 patients reported the misclassification was approximately 50\% for creatinine-based equations and still $35 \%$ for cystatin C-based equations, and equations combined creatinine and cystatin $\mathrm{C}$ were not outperform equation only based on cystatin $C$ [14]. In addition, eGFR ${ }_{C y s C}$ failed to improve the area under the curve for the diagnosis of reduced kidney function in patients with diabetes [25]. In the current 
TABLE 2: Clinical and pathological characteristics of the not/reclassified group.

\begin{tabular}{|c|c|c|c|}
\hline Variables & $\begin{array}{l}\text { Not reclassified group } \\
\qquad(n=88)\end{array}$ & $\begin{array}{l}\text { Reclassified group } \\
\quad(n=57)\end{array}$ & $P$ value \\
\hline eGFR $_{\text {CKD-EPI-Cr }}$ & $96.5 \pm 17.0$ & $76.1 \pm 11.5$ & $<0.001$ \\
\hline $\mathrm{eGFR}_{\mathrm{CKD} \text {-EPI-CysC }}$ & $79.9 \pm 17.1$ & $49.3 \pm 7.2$ & $<0.001$ \\
\hline Age (years) & $49.4 \pm 10.8$ & $51.3 \pm 8.7$ & NS \\
\hline Gender (male) (\%) & $62(70.5)$ & $38(66.7)$ & NS \\
\hline Duration of diabetes (months) & $83.4 \pm 61.6$ & $106.8 \pm 64.8$ & 0.033 \\
\hline Diabetic retinopathy (\%) & $28(32.6)$ & $22(40.0)$ & NS \\
\hline Body mass index $\left(\mathrm{kg} / \mathrm{m}^{2}\right)$ & $26.24 \pm 3.35$ & $25.67 \pm 4.59$ & NS \\
\hline Systolic blood pressure (mmHg) & $138 \pm 20$ & $144 \pm 22$ & NS \\
\hline Diastolic blood pressure (mmHg) & $86 \pm 14$ & $85 \pm 13$ & NS \\
\hline HbA1C (\%) & $7.8(6.8-9.4)$ & $7.5(6.3-10.2)$ & NS \\
\hline Initial proteinuria $(\mathrm{g} / 24 \mathrm{~h})$ & $2.4(0.8-4.8)$ & $4.2(2.3-8.8)$ & 0.001 \\
\hline Serum creatinine $(\mu \mathrm{mol} / \mathrm{L})$ & $74 \pm 18$ & $93 \pm 19$ & $<0.001$ \\
\hline Cystatin C (mg/L) & $1.02 \pm 0.15$ & $1.45 \pm 0.20$ & $<0.001$ \\
\hline Triglyceride (mmol/L) & $2.39 \pm 2.17$ & $2.03 \pm 1.61$ & NS \\
\hline Total cholesterol (mmol/L) & $4.89 \pm 1.36$ & $5.36 \pm 1.61$ & NS \\
\hline Thyroid-stimulating hormone (mU/L) & $2.93(1.79-4.09)(n=43)$ & $3.86(2.61-6.58)(n=29)$ & NS \\
\hline Free triiodothyronine $3(\mathrm{pmol} / \mathrm{L})$ & $4.41(3.92-5.10)(n=42)$ & $3.92(3.46-4.35)(n=29)$ & 0.003 \\
\hline Free triiodothyronine $4(\mathrm{pmol} / \mathrm{L})$ & $15.87(14.65-17.26)(n=42)$ & $14.54(12.50-16.66)(n=29)$ & NS \\
\hline \multicolumn{4}{|l|}{ Pathological lesions } \\
\hline Glomerular classification & & & $<0.001$ \\
\hline I & 14 & 1 & \\
\hline IIa & 39 & 14 & \\
\hline $\mathrm{IIb}$ & 6 & 7 & \\
\hline III & 26 & 29 & \\
\hline IV & 3 & 6 & \\
\hline IFTA scores & & & $<0.001$ \\
\hline 0 & 8 & 2 & \\
\hline 1 & 62 & 27 & \\
\hline 2 & 18 & 25 & \\
\hline 3 & 0 & 3 & \\
\hline Interstitial inflammation & & & $<0.001$ \\
\hline 0 & 17 & 2 & \\
\hline 1 & 70 & 45 & \\
\hline 2 & 1 & 10 & \\
\hline Arteriolar hyalinosis & & & NS \\
\hline 0 & 18 & 10 & \\
\hline 1 & 47 & 28 & \\
\hline 2 & 23 & 19 & \\
\hline Follow-up period (months) & $32 \pm 22$ & $30 \pm 18$ & NS \\
\hline Progressed to ESKD (\%) & $11(12.5)$ & $21(36.8)$ & $<0.001$ \\
\hline
\end{tabular}

Patients in the reclassified group had significantly longer diabetic duration, heavier proteinuria, and advanced pathological lesions. Data are presented as the mean \pm standard and the median with range or counts and percentages. IFTA: interstitial fibrosis and tubular atrophy; NS: not significant. A twotailed $P<0.05$ was considered statistically significant.

study, we found that cystatin C, not the creatinine, was independently associated with advanced glomerular lesions. The contradictory can be explained by the characteristics of sub- jects partially. First, different equations were compared in CKD cohorts which include various primary or secondary kidney diseases. Cystatin C may be influenced by different 

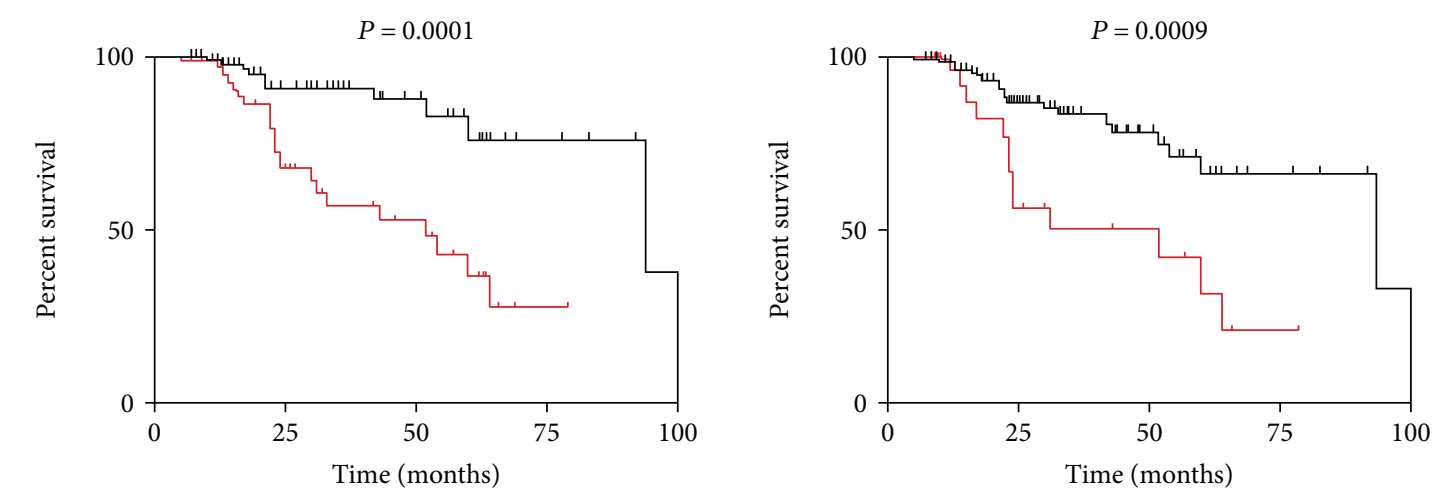

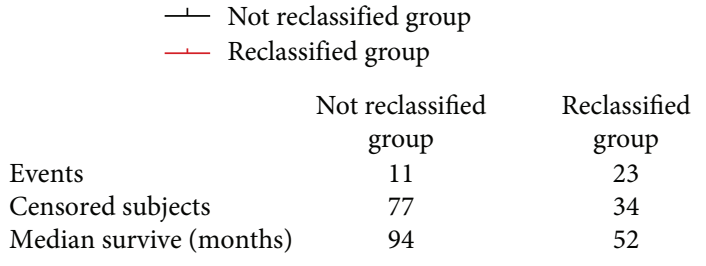

(a)

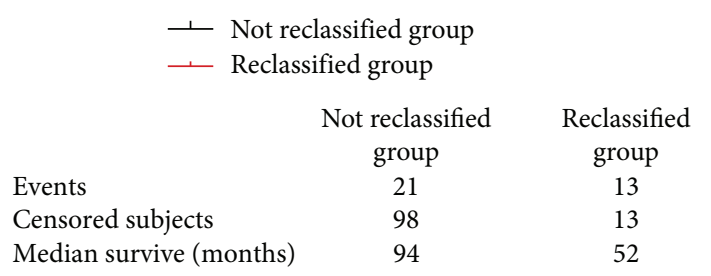

(b)

FIgURE 3: Kidney survival of patients reclassified in advanced CKD stages (3-5) by equations based on cystatin C. Comparison of kidney survival between the not reclassified group and reclassified group by eGFR $\mathrm{CKD}_{\text {-EPI-CysC }}$ (a) and eGFR $\mathrm{CKD}_{\text {-EPI-Cr-CysC }}$ (b). The kidney survival was significantly poorer by the log-rank test in the reclassified groups.

TABLE 3: Multivariable logistic regression of advanced glomerular classification and IFTA.

\begin{tabular}{|c|c|c|c|c|c|c|}
\hline \multirow{2}{*}{ Variables } & \multicolumn{3}{|c|}{ Glomerular classification of III and IV } & \multicolumn{3}{|c|}{ IFTA of 2 and 3 scores } \\
\hline & Odds ratio & $95 \% \mathrm{CI}$ & $P$ & Odds ratio & $95 \% \mathrm{CI}$ & $P$ \\
\hline Gender (male) & 0.349 & $0.174-0.700$ & 0.003 & 1.037 & $0.538-2.000$ & NS \\
\hline Age & 0.985 & $0.955-1.015$ & NS & 0.985 & $0.956-1.015$ & NS \\
\hline Systolic blood pressure & 1.008 & 0.991-1.025 & NS & 1.004 & $0.988-1.020$ & NS \\
\hline Diastolic blood pressure & 0.995 & $0.966-1.025$ & NS & 1.015 & $0.987-1.043$ & NS \\
\hline Diabetic duration & 1.003 & 0.999-1.008 & NS & 1.001 & 0.997-1.005 & NS \\
\hline Triglyceride & 0.905 & $0.769-1.063$ & NS & 0.941 & $0.803-1.104$ & NS \\
\hline Total cholesterol & 1.022 & $0.821-1.271$ & NS & 1.249 & $1.009-1.545$ & 0.041 \\
\hline Proteinuria & 1.059 & $0.979-1.146$ & NS & 1.018 & 0.949-1.092 & NS \\
\hline Serum creatinine & 1.004 & 0.993-1.015 & NS & 1.012 & $1.002-1.023$ & 0.024 \\
\hline Cystatin C & 3.771 & $1.140-12.472$ & 0.030 & 1.680 & $0.576-4.902$ & NS \\
\hline
\end{tabular}

Gender and cystatin $\mathrm{C}$ were independently associated with advanced glomerular classifications (III and IV stages); total cholesterol and serum creatinine were independently associated with higher IFTA scores. IFTA: interstitial fibrosis and tubular atrophy; CI: confidence interval; NS: not significant. A two-tailed $P<0.05$ was considered statistically significant.

disease status; even in CKD with diabetes cohort, the nondiabetic kidney disease may confound results [26, 27]. Second, subjects in previous studies were characteristic with obese (BMI $28-31 \mathrm{~kg} / \mathrm{m}^{2}$ ) and older ( $>55$ years old). Higher BMI is associated with increased fat mass which is a primary determinant of cystatin C generation [26]. And the accurate of cystatin $\mathrm{C}$ is decreased with age [28]. In the current study, the impact of age (51 years old) and BMI $\left(25.78 \mathrm{~kg} / \mathrm{m}^{2}\right)$ on the performance of $\mathrm{eGFR}_{\mathrm{CysC}}$ is limited. Third, we aimed to explore the performance of equations to detect kidney injury, not the accuracy in estimating measured GFR.

These findings suggest that $\mathrm{eGFR}_{\mathrm{CysC}}$ is more sensitive to detect kidney injury in the early stage. Therefore,
eGFR $_{\text {CysC }}$ should be considered, rather than $e G R_{C r}$ alone, for clinical decision-making, especially when eGF $\mathrm{R}_{\mathrm{Cr}}>60 \mathrm{~mL} / \mathrm{min} / 1.73 \mathrm{~m}^{2}$.

There are several limitations of the current study that should be discussed. First, the sample size was limited due to that we only enrolled patients with biopsy-proven DN. Second, all the patients were ethnic Han in Southwest China; the performance of equations may be influenced by multiethnic setting such as muscle mass and meat intake. The results should be verified in more ethnic cohorts. Third, it was a retrospective cohort study; we did not have data of measured GFR to evaluate accuracy of different equations. Fourth, creatinine and cystatin C could be fluctuated; repeated measurements should be applied. 


\section{Conclusion}

eGFR equations incorporating cystatin $\mathrm{C}$ is superior to eGFR based on creatine alone for detecting kidney injury in the early stage. The independent association between cystatin $\mathrm{C}$ and glomerular classifications might contribute to it.

\section{Data Availability}

Original data can be provided if editors require.

\section{Ethical Approval}

The protocol of study was approved by the ethics committee of West China Hospital of Sichuan University and conducted based on the principles of the Declaration of Helsinki.

\section{Consent}

Written informed consents were obtained at the time of biopsy from all the patients.

\section{Conflicts of Interest}

The authors declare that they have no competing interests.

\section{Acknowledgments}

This study was supported by Grant 81670662 from the National Natural Science Foundation of China. The Grant has supported our authors who did the work.

\section{References}

[1] H. J. Anders, T. B. Huber, B. Isermann, and M. Schiffer, "CKD in diabetes: diabetic kidney disease versus nondiabetic kidney disease," Nature Reviews Nephrology, vol. 14, no. 6, pp. 361377, 2018.

[2] M. C. Thomas, M. E. Cooper, and P. Zimmet, "Changing epidemiology of type 2 diabetes mellitus and associated chronic kidney disease," Nature Reviews Nephrology, vol. 12, no. 2, pp. 73-81, 2016.

[3] M. Afkarian, L. R. Zelnick, Y. N. Hall et al., "Clinical manifestations of kidney disease among us adults with diabetes, 19882014," JAMA, vol. 316, no. 6, p. 602, 2016.

[4] Z.-H. Liu, "Nephrology in China," Nature Reviews Nephrology, vol. 9, no. 9, pp. 523-528, 2013.

[5] G. L. Bakris and M. Molitch, "Are all patients with type 1 diabetes destined for dialysis if they live long enough? Probably not," Diabetes Care, vol. 41, no. 3, pp. 389-390, 2018.

[6] K. Matsushita, B. K. Mahmoodi, M. Woodward et al., "Comparison of risk prediction using the CKD-EPI equation and the MDRD study equation for estimated glomerular filtration rate," Journal of the American Medical Association, vol. 307, no. 18, 2012.

[7] Kidney Disease: Improving Global Outcomes (KDIGO) CKD Work Group, "KDIGO 2017 clinical practice guideline update for the diagnosis, evaluation, prevention, and treatment of chronic kidney disease-mineral and bone disorder (CKDMBD)," Kidney International Supplements, vol. 7, no. 1, pp. 1-59, 2017.
[8] American Diabetes Association, "Classification and diagnosis of diabetes: standards of medical care in diabetes 2018," Diabetes Care, vol. 41, Supplement 1, pp. S13-S27, 2018.

[9] T. W. C. Tervaert, A. L. Mooyaart, K. Amann et al., "Pathologic classification of diabetic nephropathy," Journal of the American Society of Nephrology, vol. 21, no. 4, pp. 556-563, 2010.

[10] S. Nair, S. V. O'Brien, K. Hayden et al., "Effect of a cooked meat meal on serum creatinine and estimated glomerular filtration rate in diabetes-related kidney disease," Diabetes Care, vol. 37, no. 2, pp. 483-487, 2014.

[11] A. Levin and P. E. Stevens, "Summary of KDIGO 2012 CKD guideline: behind the scenes, need for guidance, and a framework for moving forward," Kidney International, vol. 85, no. 1, pp. 49-61, 2014.

[12] National Kidney Foundation, "K/DOQI clinical practice guidelines for chronic kidney disease: evaluation, classification, and stratification," American Journal of Kidney Diseases, vol. 39, no. 2, Supplement 1, pp. S1-S266, 2002.

[13] S. M. Doshi and A. N. Friedman, "Diagnosis and management of type 2 diabetic kidney disease," Clinical Journal of the American Society of Nephrology, vol. 12, no. 8, pp. 1366-1373, 2017.

[14] S. Luis-Lima, B. Escamilla-Cabrera, N. Negrín-Mena et al., "Chronic kidney disease staging with cystatin C or creatinine-based formulas: flipping the coin," Nephrology Dialysis Transplantation, vol. 34, no. 2, pp. 287-294, 2018.

[15] H. Pottel, P. Delanaye, E. Schaeffner et al., "Estimating glomerular filtration rate for the full age spectrum from serum creatinine and cystatin C," Nephrology Dialysis Transplantation, vol. 32, no. 3, pp. 497-507, 2017.

[16] A. D. Rule and W. K. Kremers, "What is the correct approach for comparing GFR by different methods across levels of GFR?," Clinical Journal of the American Society of Nephrology, vol. 11, no. 9, pp. 1518-1521, 2016.

[17] R. D. Perrone, N. E. Madias, and A. S. Levey, "Serum creatinine as an index of renal function: new insights into old concepts," Clinical Chemistry, vol. 38, no. 10, pp. 1933-1953, 1992.

[18] E. A. Oliveira, W. W. Cheung, K. G. Toma, and R. H. Mak, "Muscle wasting in chronic kidney disease," Pediatric Nephrology, vol. 33, no. 5, pp. 789-798, 2018.

[19] S. B. Heymsfield, C. Arteaga, C. McManus, J. Smith, and S. Moffitt, "Measurement of muscle mass in humans: validity of the 24-hour urinary creatinine method," The American Journal of Clinical Nutrition, vol. 37, no. 3, pp. 478-494, 1983.

[20] C.-W. Tsai, M. E. Grams, L. A. Inker, J. Coresh, and E. Selvin, "Cystatin C- and creatinine-based estimated glomerular filtration rate, vascular disease, and mortality in persons with diabetes in the U.S.," Diabetes Care, vol. 37, no. 4, pp. 1002-1008, 2014.

[21] F. Gaspari, P. Ruggenenti, E. Porrini et al., "The GFR and GFR decline cannot be accurately estimated in type 2 diabetics," Kidney International, vol. 84, no. 1, pp. 164-173, 2013.

[22] A. D. Rule and R. J. Glassock, "GFR estimating equations: getting closer to the truth?," Clinical Journal of the American Society of Nephrology, vol. 8, no. 8, pp. 1414-1420, 2013.

[23] M. E. Grams, S. P. Juraschek, E. Selvin et al., "Trends in the prevalence of reduced GFR in the United States: a comparison of creatinine- and cystatin C-based estimates," American Journal of Kidney Diseases, vol. 62, no. 2, pp. 253-260, 2013. 
[24] R. J. MacIsaac, E. I. Ekinci, E. Premaratne et al., "The chronic kidney disease-epidemiology collaboration (CKD-EPI) equation does not improve the underestimation of glomerular filtration rate (GFR) in people with diabetes and preserved renal function," BMC Nephrology, vol. 16, no. 1, article 198, 2015.

[25] F. Iliadis, T. Didangelos, A. Ntemka et al., "Glomerular filtration rate estimation in patients with type 2 diabetes: creatinine- or cystatin C-based equations?," Diabetologia, vol. 54, no. 12, pp. 2987-2994, 2011.

[26] S. Lemoine, F. Guebre-Egziabher, F. Sens et al., "Accuracy of GFR estimation in obese patients," Clinical Journal of the American Society of Nephrology, vol. 9, no. 4, pp. 720-727, 2014.

[27] Y. Qi, P. Hu, Y. Xie et al., "Glomerular filtration rate measured by ${ }^{99 \mathrm{~m}}$ Tc-DTPA renal dynamic imaging is significantly lower than that estimated by the CKD-EPI equation in horseshoe kidney patients," Nephrology, vol. 21, no. 6, pp. 499-505, 2016.

[28] L. Fan, L. A. Inker, J. Rossert et al., "Glomerular filtration rate estimation using cystatin $\mathrm{C}$ alone or combined with creatinine as a confirmatory test," Nephrology Dialysis Transplantation, vol. 29, no. 6, pp. 1195-1203, 2014. 


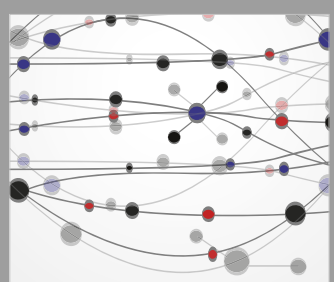

The Scientific World Journal
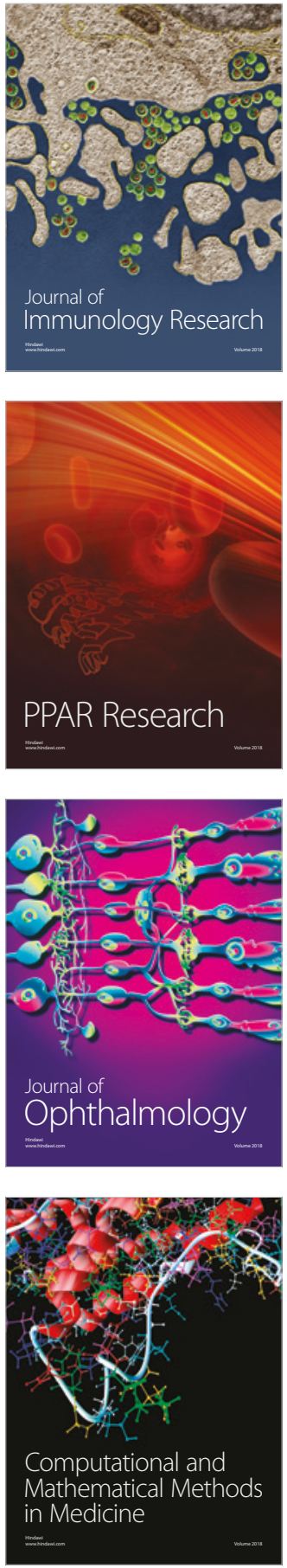

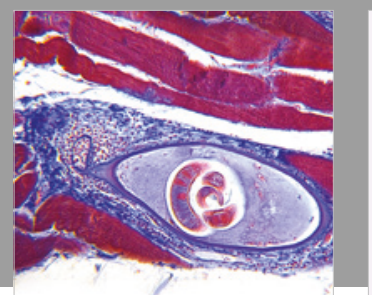

Gastroenterology Research and Practice

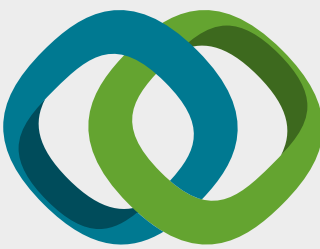

\section{Hindawi}

Submit your manuscripts at

www.hindawi.com
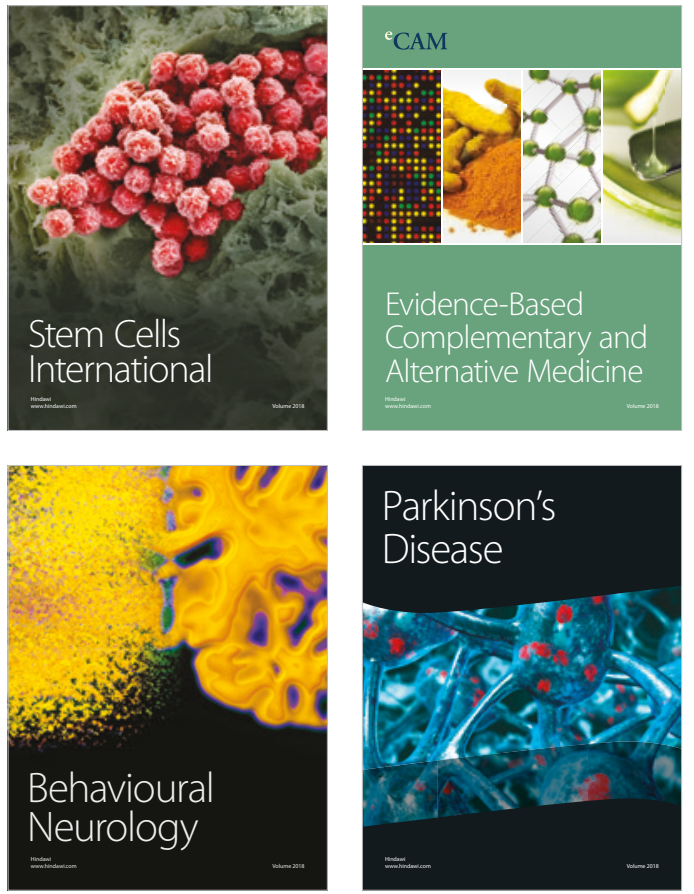

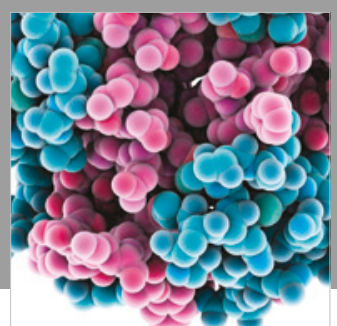

ournal of

Diabetes Research

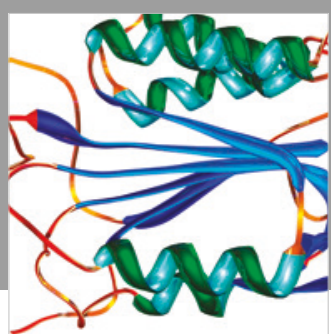

Disease Markers
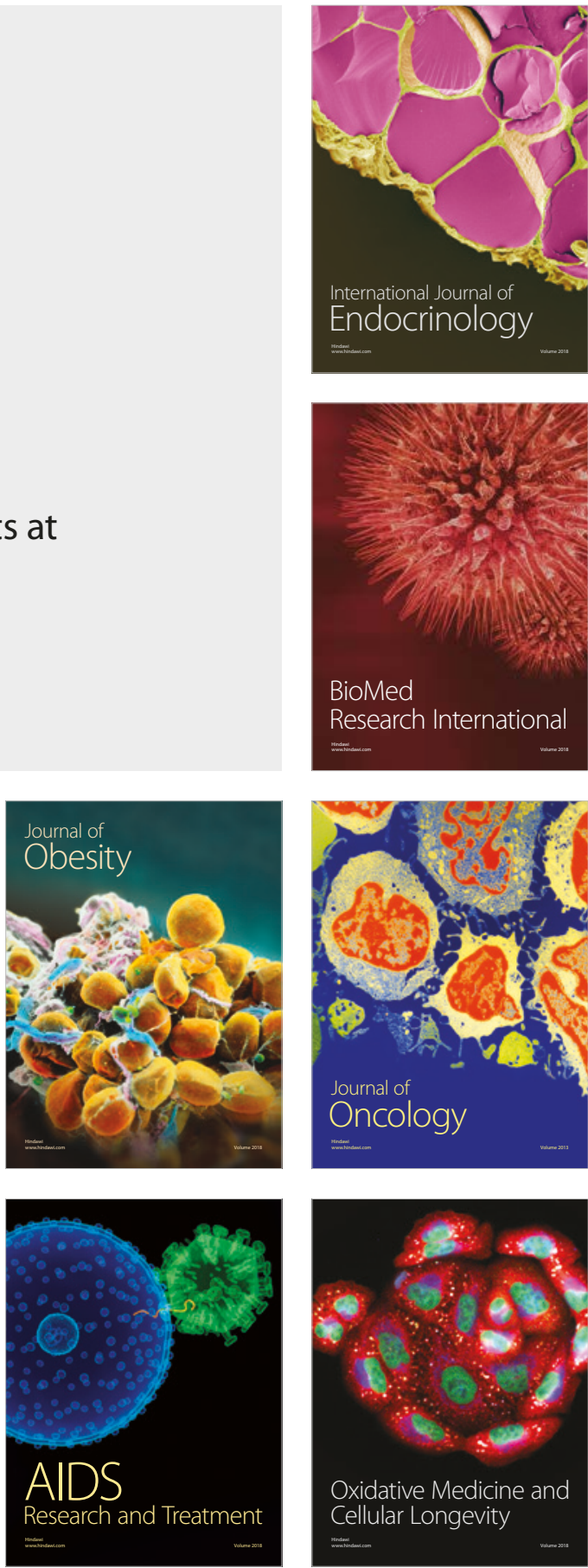